Conclusion This pilot study may suggest the presence of sufficient pertussis seroimmunity rates in the studied mothers-neonates pair. Still, there were some failures in immune acquisition probably due to waning of immunity with age. Transplacental passage of pertussis antibodies may not confer similar seroimmunity to pertussis in neonates as in mothers. Wider scale studies would allow better insight into the pertussis immune status of the females in the child bearing period in our country and hence the need for booster immunization during pregnancy.

\section{G610(P) RETROSPECTIVE ANALYSIS OF NEONATAL DEATHS SECONDARY TO INFECTIONS IN ENGLAND AND WALES, 2013-2015}

'L Ahmed, 'GI Oligbu, 'L Ferraras, 1,2 $\mathrm{SN}$ Ladhani. 'Paediatric Infectious Diseases Research Group, St. George's University of London, London, UK; ${ }^{2}$ Immunisation and Countermeasures Division, Public Health England, London, UK

\subsection{6/archdischild-2020-rcpch.524}

Purpose To estimate the overall and infection related neonatal mortality rate and the pathogens responsible using electronic death registrations.

What is already known on this topic

- The UK has one of the highest neonatal mortality rates among industrialised countries.

- Infections are associated with significant morbidity and mortality in neonates.

- Neonatal mortality rates have declined with advancements in neonatal care.

Results Total number of deaths secondary to infection per gestation. Early onset ( $<7$ days), Late onset $>7$ days. ${ }^{1}$

The most common pathogens responsible for neonatal mortality in each category. Gram positive: GBS ( $10 \%)$, gram negative: E.Coli $(\sim 6 \%)$, viruses: HSV- 3\%, Fungi: candida $1 \% .^{1}$

- Design: retrospective analysis of national electronic death registrations data

- Setting : England and Wales

- Patients: Neonates aged $<28$ days

- Main outcome measures: Overall and infection related mortality rate per 1,000 live births in term, preterm (28 36 weeks) and extremely preterm ( $<28$ weeks) neonates the contribution of infections and specific pathogens; comparison withmortality rates in 200305 .

Conclusion Overall and infection related neonatal mortality rates have declined, but the contribution of infection and of specific pathogens has not changed.

What this study adds

- Both the overall and infection related neonatal mortality rates have declined over the past decade.

- The contribution of infections and of specific pathogens to neonatal deaths has not changed.

- Group B Streptococcus remains the single most important pathogen associated with neonatal deaths.

\section{REFERENCE}

1. Office for National Statistics. Vital Statitistics Summary, England and Wales. Health Stat Q. 2009:43:26.

\section{G611(P) SUBSIDIZED MALARIA PROPHYLAXIS FOR CHILDREN: IS} IT USEFUL?

C Pelly, K Jewell, J Lawrence, D Hutchens, S Johnson. Lewisham and Greenwich NHS Trust, NHS, London, UK

\subsection{6/archdischild-2020-rcpch.525}

Aim To evaluate whether a borough-wide policy change to remove subsidisation of antimalarials affected paediatric malaria rates.

Background Children are more susceptible to acquiring severe malaria and often have a delayed diagnosis in the UK. Following trends in international travel, rates of imported malaria are not diminishing in the UK, despite the existence of effective malaria prophylaxis medications. Malaria commonly affects those who are travelling to visit friends and relations (VFR); for whom multifaceted barriers to effective prophylaxis have been identified. In 2005 a nationwide policy was introduced to stop subsidiziation of malaria prophylaxis drugs. Some boroughs did not implement this due to large populations of VFRs. In October 2017 one borough updated their policy to remove subsidies, in concordance with national policy (and the adjacent 'control' borough).

Method This retrospective analysis reviewed all paediatric patients ( $<16$ years) diagnosed with malaria in the 12 months prior and post local policy change. A comparison was made with the control hospital over the same time-period. Malaria cases were diagnosed using thick and thin smear microscopy. Medical records were reviewed to assess prophylaxis use and adherence.

Results In the hospital with a recent change in policy there was no increase in patients with malaria after the subsidy was removed; in year 1,7 patients tested positive out of 61 screens (16\% positive test rate), compared to 6 positive cases in year $2(8 \%$ positive test rate). In the control hospital there were 10 positive cases in year 1 (12\% positive test rate), and 3 cases in year 2 ( $3 \%$ positive test rate). 14/29 positive patients had prophylaxis prescribed. Poor adherence to prophylaxis was reported in $13 / 14$ of these cases.

Conclusion These findings demonstrate cases of paediatric malaria fell over the study period in both hospitals. It seems prophylaxis subsidization is unlikely to account for this. This study supports prophylaxis is often not taken as prescribed amongst positive malaria cases. However, it did not add weight to the argument that subsidies prevent malaria. Rather strategic interventions encompassing the spectrum of barriers to effective prophylaxis are required to improve children's access to prophylaxis and prevent children becoming ill with malaria.

\section{G612(P) ENTEROVIRUS D68 REAL-TIME 2-STEP PCR: A USEFUL DIAGNOSTIC TOOL IN LOWER RESPIRATORY TRACT INFECTIONS IN CHILDREN?}

${ }^{1} \mathrm{P}$ Makiello, ${ }^{2} \mathrm{~A}$ Vossen, ${ }^{2} \mathrm{E}$ Wessels. 'Internal Medicine, Dumfries and Galloway Royal Infirmary, Dumfries, UK; ${ }^{2}$ Medical Microbiology, Leiden University Medical Centre, Leiden, The Netherlands

\subsection{6/archdischild-2020-rcpch.526}

Introduction EV-D68 is likely under-reported because an enterovirus PCR is usually not run for patients with respiratory symptoms; moreover $73 \%$ of rhinovirus (HRV) assays detect EV-D68, misleading clinicians as to the causative virus. 